\title{
GO-modified flexible polymer nanocomposites fabricated via 3D stereolithography
}

\author{
Chi Him Alpha Tsang $(\varangle)^{1,2 *}$, Adilet Zhakeyev ${ }^{3 *}$, Dennis Y.C. Leung $(\varangle)^{2}$, Jin Xuan $(\varangle)^{3}$ \\ 1 School of Environmental Sciences and Engineering, Sun Yat-Sen University, Guangzhou 510006, China \\ 2 Department of Mechanical Engineering, The University of Hong Kong, Hong Kong, China \\ 3 Department of Chemical Engineering, Loughborough University, Loughborough, LE11 3TU, UK
}

(C) The Author(s) 2019. This article is published with open access at link.springer.com and journal.hep.com.cn 2019

\begin{abstract}
Graphene oxide (GO) induced enhancement of elastomer properties showed a great deal of potential in recent years, but it is still limited by the barrier of the complicated synthesis processes. Stereolithography (SLA), used in fabrication of thermosets and very recently in "flexible" polymers with elastomeric properties, presents itself as simple and user-friendly method for integration of GO into elastomers. In this work, it was first time demonstrated that GO loadings can be incorporated into commercial flexible photopolymer resins to successfully fabricate $\mathrm{GO} /$ elastomer nanocomposites via readily accessible, consumer-oriented SLA printer. The material properties of the resulting polymer was characterized and tested. The mechanical strength, stiffness, and the elongation of the resulting polymer decreased with the addition of GO. The thermal properties were also adversely affected upon the increase in the GO content based on differential scanning calorimetry and thermogravimetric analysis results. It was proposed that the GO agglomerates within the $3 \mathrm{D}$ printed composites, can result in significant change in both mechanical and thermal properties of the resulting nanocomposites. This study demonstrated the possibility for the development of the GO/elastomer nanocomposites after the optimization of the GO/"flexible" photoreactive resin formulation for SLA with suitable annealing process of the composite in future.
\end{abstract}

Keywords graphene oxide, polymer, flexible, 3D printing, stereolithography

Received November 1, 2018; accepted March 2, 2019

E-mails: chtsang2006@yahoo.com.hk (Tsang C), ycleung@hku.hk (Leung D), j.xuan@lboro.ac.uk (Xuan J)

${ }^{*}$ These authors contributed equally to this work.

\section{Introduction}

Recently, 3D printing technology has attracted interest of different research groups, since it is a useful technology in the high-end machine sector [1]. However, 3D printed polymers suffer from weak mechanical properties, which restricts the application of such materials [1]. The addition of foreign materials, such as nanoparticles, to polymers can improve their mechanical, optical, and thermal properties [1]. Due to the attractive properties of graphene, such as high mechanical strength [2], and high electrical [3] and thermal conductivity [4], it recently became a popular material for research to improve the properties of polymer in different aspects. Graphene oxide (GO) reinforcement of $3 \mathrm{D}$ printing polymers became a new focus in the field. Two typical practices for such process include in-situ intercalation [5-7], and the solution intercalation $[1,5,6]$. Among these methods, direct dispersion of GO into polymer through solution intercalation $[5,6]$ was one of the most convenient ways for the 3D structural printing of graphene based materials/polymer matrix, since the in-situ intercalation of GO with polymer requires an extra mechanical process in order to mix the GO and polymer homogeneously, especially when GO is obtained from a freeze drying process and not in a powder form. In practice, water, acetone, benzene family, tetrahydrofuran, formaldehyde, and chloroform are the commonly used solvents for GO dispersion [5,6]. Liquid monomer resins usually have a good solubility in most organic solvents. However, different kinds of resins vary in stability towards the solvent in solution intercalation process as it may cause structural destruction in the final product when incorrect solvent is used. As a result, correct combination of the solvent and the resin for the GO reinforced 3D structural polymer production becomes an important factor.

Elastomers are polymers that show a viscoelastic behavior, with low Young's modulus, high failure strain 
and weak intermolecular interaction compared to other polymers. These properties made them exhibited rubberlike elasticity $[8,9]$. Some of the commonly studied elastomers included polyvinylidene fluoride (PVDF) [10], polyurethanes [9,11], styrene-butadiene rubber [12] and natural rubber latex or natural rubber $[9,13]$. In current research, GO modified elastomer has been developed by different groups [8-15], they were mainly synthesized by traditional methods such as melt mixing [8], solution/latex blending $[8,9,11,12]$, in-situ polymerization [8], and film processing $[10,13]$. The GO modified elastomers showed stronger mechanical properties than the corresponding pure elastomers $[13,15]$. However, complicated synthesize process is the major drawback of these methods. As a result, development of the $\mathrm{GO} /$ elastomers synthesis with simple and friendly method became a new trend, including the use of 3D printing technology. Up to date, the GO modified 3D printed thermosets using photopolymer resins via stereolithography (SLA) have been demonstrated by some research groups [1,7], but the process has not been optimized yet. Moreover, all the previous attempts used thermosets [1,7]. Even though some of the groups demonstrated recently the use of GO in the SLA, the resin used was also hard type in nature [16]. In practice, flexible 3D printed materials can be more useful than the hard ones in some particular application, such as robotics and medical devices. There are a few reports detailing the marriage between $\mathrm{GO}$ coatings and 3D printed elastomers resulting in a practical application [14]. However, none of them involve direct $3 \mathrm{D}$ printing of $\mathrm{GO} /$ commercial elastomer composites. There are several important considerations involved in direct photopolymerization of $\mathrm{GO} /$ elastomer composites via SLA. In order to synthesize GO modified 3D printed soft-polymers or elastomers for practical purposes, the choice of correct solvent for the GO dispersion in the solution-intercalation step of the SLA monomer gel is very important, in order to prevent the degradation of a photopolymer resin by the solvent [1]. More importantly, some group attempted to synthesize the stable GO/thermoset resin for SLA recently, but it involved the chemical functionalization of GO prior the mixing with the photopolymer resin. Furthermore, the resin itself also required pre-treatment with a number of chemicals [17]. Such processes make GO/thermoset resin synthesis not as user-friendly and readily accessible. More importantly, the fabrication of $\mathrm{GO} /$ elastomer composites via SLA process has not yet been reported. This provides a window of opportunity to dwell into the possibility of developing GO modified flexible 3D printed materials, in a more user friendly and readily accessible manner.

Commercially available elastomer (or "flexible") photoresins such as Formlabs flexible and Spot-E Elastic exhibit limited elongations ranging from $90 \%$ to $100 \%$ [18]. It was previously demonstrated that incorporation of GO into commercial PIC 100 resin (EnvisionTec), which is used to $3 \mathrm{D}$ print rigid plastics, can result in increased tensile strength as well as increased elongation at break [1]. The objective of this particular work was to study the effects of GO addition into a commercially available SLA elastomer resin to fabricate $\mathrm{GO} /$ elastomer nanocomposites via readily accessible, consumer oriented SLA printer in a userfriendly manner. It was demonstrated that the dispersion of $\mathrm{GO}$ in the resin played an important role on mechanical and thermal properties of the $3 \mathrm{D}$ printed products.

\section{Materials and methods}

\subsection{Materials}

The following materials and chemicals were used directly as purchased: Graphite powder (325 mesh, Uni-Chem), $\mathrm{P}_{2} \mathrm{O}_{5}$ (99\%, Acros-organic), $\mathrm{K}_{2} \mathrm{~S}_{2} \mathrm{O}_{8}$ (99\%, Fisher Scientific), $\mathrm{KMnO}_{4}$ (99\%, Sigma-Aldrich), $\mathrm{H}_{2} \mathrm{O}_{2}$ (30\%, SigmaAldrich), sulphuric acid (98\%, Sigma-Aldrich), hydrochloric acid (37\%, Sigma-Aldrich), chloroform (AcroOrganic, 99\%), isopropanol (Acro-Organic, 99\%) and photoreactive resin composed of mixture of acrylated oligomers, acrylated monomers and photoinitiator (Formlabs flexible Resin, FLFLGR01), which is the SLA resin candidate used for the flexible polymer production in this study.

\subsection{GO/elastomer nanocomposite fabrication via SLA}

\subsubsection{Raw GO preparation}

The raw GO was synthesized from the modified Hummer's method which was reported elsewhere [19]. Briefly, $2 \mathrm{~g}$ of graphite powder $1 \mathrm{~g} \mathrm{~K}_{2} \mathrm{~S}_{2} \mathrm{O}_{8}$ and $1 \mathrm{~g} \mathrm{P}_{2} \mathrm{O}_{5}$ was added to a $50 \mathrm{~mL} \mathrm{98 \%} \mathrm{sulphuric} \mathrm{acid.} \mathrm{The} \mathrm{mixtures} \mathrm{were} \mathrm{stirred} \mathrm{for}$ $6 \mathrm{~h}$ to ensure complete mixing. The resulting solution was then rinsed and centrifuged by DI water until the $\mathrm{pH}$ of the pure mixture became neutral $(\mathrm{pH}=7)$. The product was then dried in an oven at $80^{\circ} \mathrm{C}$ overnight to remove the residual water. The dried residue (preoxidized graphite oxide) was cracked into powder.

The preoxidized graphite oxide powder was then put into a cold $\left(5^{\circ} \mathrm{C}-10^{\circ} \mathrm{C}\right)$ sulphuric acid $(98 \%)$ solution for mixing, followed by a slow and stepwise addition of $6 \mathrm{~g}$ of $\mathrm{KMnO}_{4}$ powder. After adding all the $\mathrm{KMnO}_{4}$ powder into the mixture solution, the system temperature was then increased and maintained at $35^{\circ} \mathrm{C}$ for $2 \mathrm{~h}$ in order to achieve the second step oxidation of the graphite oxide powder into graphene oxide. The system was quenched by the addition of $30 \% \mathrm{H}_{2} \mathrm{O}_{2}(10 \mathrm{~mL})$ within $15 \mathrm{~min}$, followed by the addition of $250 \mathrm{~mL}$ DI water for rinsing and neutralizing the solution. The rinsed solution was further cleaned by $10 \mathrm{v} / \mathrm{v} \% \mathrm{HCl}$ with centrifuge for a few times to remove the residual metal ions in the raw GO suspension. The acid cleaned raw GO was then rinsed by DI water with 
centrifuge to neutralize the solution $\mathrm{pH}$ to around $4-5$. The rinsed $\mathrm{GO}$ was then further purified in dialysis tubing for 2-3 weeks with DI water for further removal of residual $\mathrm{HCl}$ in the mixture. The cleaned raw GO was finally freeze dried by a freeze-drying system to remove all the water in the raw GO suspension. The spongy as-prepared GO block was then directly used without further treatment.

\subsubsection{D printing process}

3D printed GO nanocomposites were fabricated through the method reported in the literature [1]. Briefly, $0.8 \mathrm{~g}$ of the as-prepared GO blocks were dispersed into $120 \mathrm{~mL}$ of chloroform solution in an ultrasonic bath for approximately $1 \mathrm{~h}$ to obtain an organic GO dispersion. Resin comprising of mixture of acrylated monomers and acrylated oligomers ( $160 \mathrm{~g}$ ) were then added to the organic GO dispersion, followed by further ultrasonic mixing for another $2 \mathrm{~h}$. The resulting mixture was then stirred under heating at $95^{\circ} \mathrm{C}$ for $18 \mathrm{~h}$ for the removal of the organic solvents to obtain a pure $0.5 \mathrm{wt} \%$ GO-resin mixture. Generally, the presence of GO can considerably affect the photorosslinking behavior by preventing the penetration of light into the resin. The addition of GO to a photopolymer resin results in decrease in the penetration depth of the resin [7]. The penetration depth $\left(D_{\mathrm{p}}\right)$, defines the depth of the resin at which the light intensity is reduced to $1 / \mathrm{e}$ of its value on the resin surface. The higher is the GO content the lower the penetration depth. Since the minimum layer thickness of Form $1+3 \mathrm{D}$ printer is $25 \mathrm{um}$, as well as the fact that at the time Formlabs printers had a closed material system (i.e., not possible to change energy exposure density parameters, such as laser scanning speed, laser intensity and number of passes per layer), the concentrations of GO above $0.3 \mathrm{wt}-\%$ resulted in cured layers not being fully attached to the build platform, at the printer settings for flexible resin. Therefore, the GO loading in this work were set to be $0.1-0.3$ wt- $\%$ GO. The $0.1-0.3$ wt- $\%$ $\mathrm{GO} /$ polymer resin was prepared by dilution of the $0.5 \mathrm{wt}-\%$ resin mixture by a blank resin/chloroform mixture prepared with the same procedure mentioned above.

The resulting mixture was then transferred to the resin tank of the SLA 3D printer (Form 1+, Formlabs Inc.) after a short time of cooling under room temperature. Fabrication of 3D structure was achieved via a bottom-up SLA approach. The as-prepared printed structure was washed in an isopropanol solution for a few minutes in order to remove the residual resin on the product surface. The dry parts were then further hardened by an UV-light source, followed by a polishing process. For comparison, two pure resin 3D structures were also prepared, with one of them containing no GO and no pre-treatment steps, while another containing no GO but was further treated with chloroform with the steps similar to the $\mathrm{GO} /$ resin mixture processing as mentioned above.

\subsection{Characterization}

The surface morphology of the 3D printed GO nanocomposites were characterized by scanning electron microscopy (SEM) (Hitachi S4800) and transmittance electron microscopy (TEM) (Philips FEI Tecnai G2 20 S-Twin Scanning TEM) system. The elemental information of the raw GO/polymer resin mixture was analyzed by Frontier IR spectrometer (FTIR, PerkinElmer). While the phase structure of the $3 \mathrm{D}$ printed $\mathrm{GO}$ nanocomposites was analyzed by the differential scanning calorimetry (DSC 2010, TA Instruments) and thermogravimetric analysis (TGA Q500), respectively.

\subsection{Mechanical test}

The mechanical properties of 3D printed GO nanocomposites were assessed under uniaxial tension at room temperature using a mechanical testing system (Mach-1, Biomomentum) with a $10 \mathrm{~kg}$ load cell to determine the change in material properties of the flexible polymer with respect to the change in GO content $(0,0.1,0.2$ and $0.3 \mathrm{wt}-\%)$. Tensile test specimens were $3 \mathrm{D}$ printed in the form of standard dumbbell-shape, with the tensile test parameters in accordance with the Standard Test Method for Tensile Properties of Plastics (ASTM D638-14), the build orientation during the SLA process was kept constant for test specimens with different GO content. Tensile stress was calculated as the measured force normalised to the cross sectional area of the sample, whereas the applied strain was measured as displacement normalised to the gauge length of the sample. Young's Modulus was calculated as the slope of the normalised stress-strain curve using linear regression of the linear region of the curve.

\section{Results and discussion}

The digital image of the 3D printed blocks prepared with the flexible resin, with different GO content $(0-0.3 \mathrm{wt}-\%)$, is shown in Fig. 1. We observed that the blank flexible polymers had a semi-transparent color in appearance no

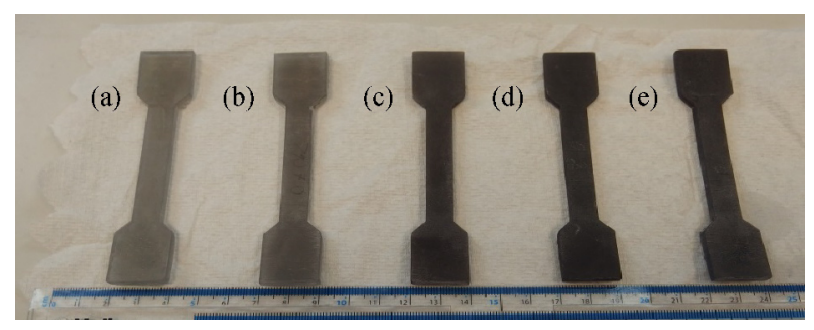

Fig. 1 Digital image of the 3D structure from pure polymer and $\mathrm{GO} /$ Formlabs flexible nanocomposite with different GO concentrations: (a) 0 wt- $\%$ GO; (b) 0 wt- $\%$ GO after $\mathrm{CH}_{3} \mathrm{Cl}$ evaporation; (c) $0.1 \mathrm{wt}-\% \mathrm{GO}$; (d) $0.2 \mathrm{wt}-\% \mathrm{GO}$; (e) $0.3 \mathrm{wt}-\%$ GO. 
matter whether the raw resin was treated with chloroform or not. In comparison to the blank products, in the GOmodified SLA printed flexible polymer nanocomposites, the color of the products was changed from semitransparent to dark green after the raw GO was mixed with the resin. It was similar to the appearance of SLA fabricated GO nanocomposites, when other photopolymer resin formulation was used as reported elsewhere [1]. The appearance of the resulting structure became darker when the amount of GO added to the resin increased from 0.1 to $0.3 \mathrm{wt}-\%$. For the nanocomposite formulation synthesis, resin quality check to determine the degree of GO nanosheets dispersion in the resin gel was an important factor for the SLA process involving the use of GO as nanofiller material. TEM images presented in Figs. 2 and $3(\mathrm{a}, \mathrm{b})$ represent $0.1 \mathrm{wt} \% \mathrm{GO} /$ Formlabs flexible resin and raw GO dispersed in chloroform respectively. From Fig. 2(d) it can be observed that GO nanosheet flakes tend to agglomerate in the Formlabs' flexible resin, even after ultrasonication and mechanical stirring steps. The extent of agglomeration ranges from 10 to $200 \mathrm{~nm}$, for instance Figs. 2(a $\left(\mathrm{a}^{-} \mathrm{c}\right)$, illustrate $\mathrm{a} \sim 200 \mathrm{~nm}$ GO agglomerate in the SLA resin. As a comparison, Figs. 3(c,d) show the TEM and SEM images of the raw GO after dispersing in chloroform and water respectively, which demonstrates a clear nanosheet structure without denaturing. The average size of the GO nanosheets in raw GO according to Fig. 3(d) was approximately $0.57-5.3 \mu \mathrm{m}$ in size. Such finding showed that the aggregation of the GO nanosheets does not take place in the chloroform throughout the dispersion step, but aggregation is clearly present after GO nanosheets incorporation into photopolymer resin formulation (Fig. 2) when compared to the SEM image of raw GO

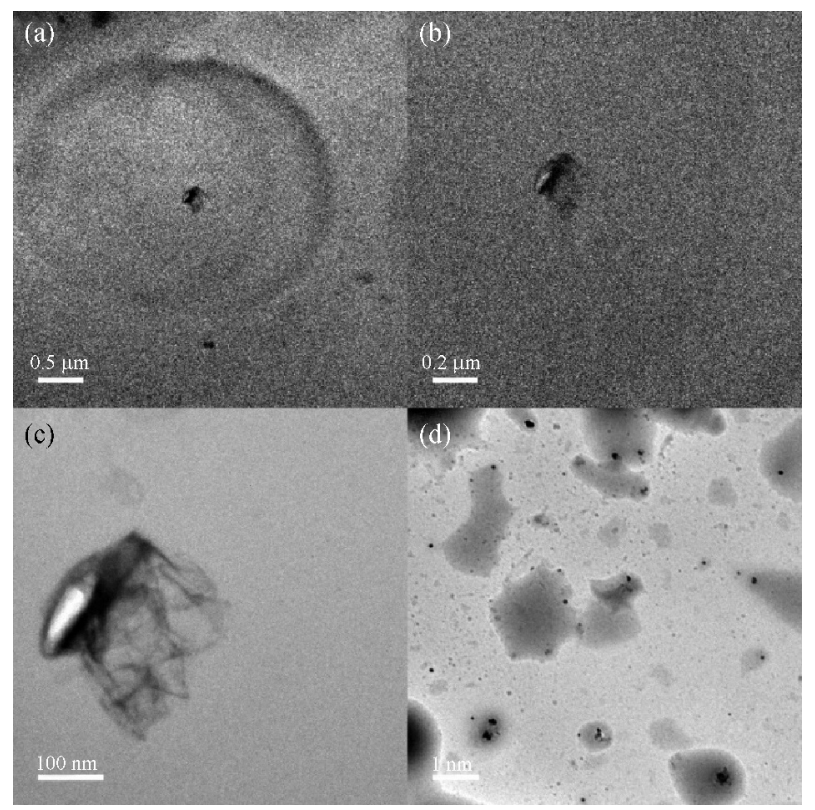

Fig. 2 TEM image of 0.1 wt-\% GO/MA raw gel (Scale bar: (a) $0.5 \mu \mathrm{m}$, (b) $0.2 \mu \mathrm{m}$, (c) $100 \mathrm{~nm}$, and (d) $1 \mu \mathrm{m}$ ).

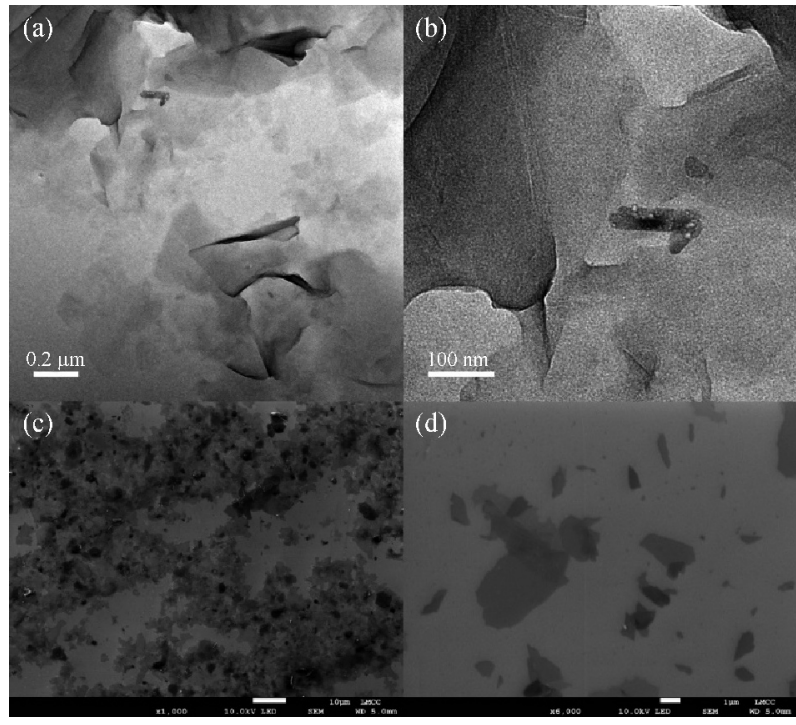

Fig. 3 (a) and (b) TEM images of raw GO dispersed in chloroform under different magnification; (c,d) corresponding SEM images (Scale bar: (a) $0.2 \mu \mathrm{m}$, (b) $100 \mathrm{~nm}$, (c) $10 \mu \mathrm{m}$, (d) $1 \mu \mathrm{m})$.

nanosheets (Figs. 3(c,d)) and optical microscope image of $\mathrm{GO}$ in the $0.2 \mathrm{wt} \% \mathrm{GO} /$ flexible polymer composite (Fig. S1, cf. Electronic Supplementary Material). It showed that raw GO nanosheet showed clear sheet structure, but the high degree of aggregation was observed in all 3D printed GO composites, particularly in the 0.2 wt- $\%$ sample as illustrated in Fig. S1. Poor affinity between GO and the resin results in aggregation of GO. Increase in GO loading might lead to excessive interplatelet interaction, as opposed to GO-resin hydrogen bonding, which in turn decreases GO-resin interaction leading to aggregation. Such phenomenon can result in poor homogeneity even after the mechanical mixing, ultrasonication, sheer mixing or combination of thereof had been applied. GO agglomeration was also indirectly confirmed in mechanical studies analysis of SLA printed GO nanocomposites, which will be further discussed in more detail.

The morphology of the SLA printed GO/Formlabs flexible nanocomposite with different GO content observed via SEM (Figs. 4 and S2) was similar to that of GO-poly(ethylenglycol)diacrylate (GO-PEGDA) from the work carried out by Chiappone et al. [20]. on the GOPEGDA synthesis by digital light processing (DLP). The surface morphology characterization by SEM (Figs. 4 and S2) showed that the cross-section morphology of the representative product of $0.1 \mathrm{wt}-\% \mathrm{GO} / \mathrm{resin}$ showed a typical brittle fracture, and the fracture surface was relatively smooth. However, higher degree of aggregation was observed in the optical microscope image of $0.2 \mathrm{wt}-\%$ $\mathrm{GO} /$ flexible polymer composite under UV-light as shown in Fig. S1. Such result showed that the flat GO nanosheets (Fig. 3) were aggregated in the SLA resin throughout the 


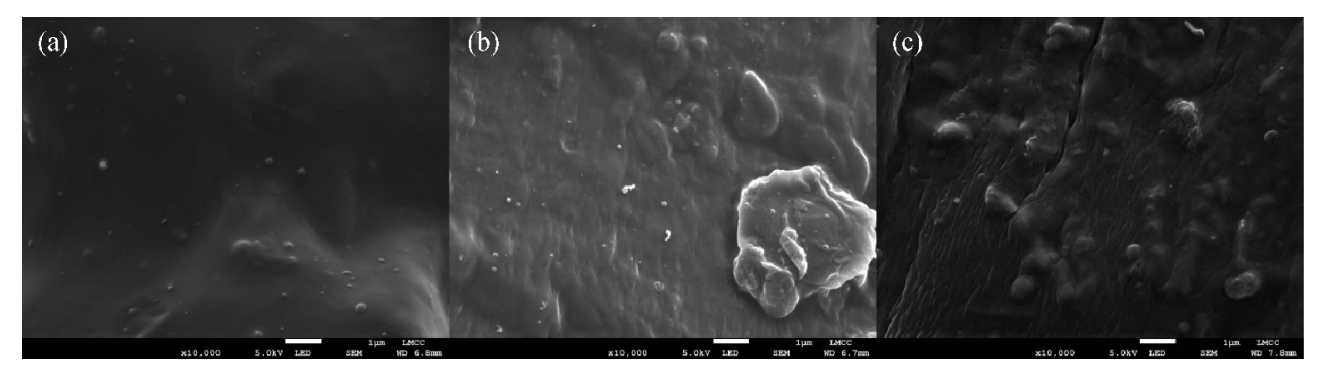

Fig. 4 SEM image of (a) $0.1 \mathrm{wt}-\%$, (b) $0.2 \mathrm{wt}-\%$, and (c) $0.3 \mathrm{wt}-\% \mathrm{GO} /$ Formlabs flexible nanocomposite (Scale bar: $1 \mu \mathrm{m}$ ).

3D printing process.

Figure S3 demonstrates the FTIR of the GO/Formlabs flexible composite with $0.1 \mathrm{wt}-\% \mathrm{GO}$. It showed that there was almost no obvious difference between the 3D printed GO/Formlabs flexible nanocomposite and the blank Formlabs flexible polymer. To determine the effect of the addition of $\mathrm{GO}$ on the thermal properties of the $3 \mathrm{D}$ printed composites, their thermal behavior was determined using DSC and TGA. Based on the DSC results (Fig. 5 and Table 1), the glass transition temperature $\left(T_{\mathrm{g}}\right)$ of the $3 \mathrm{D}$ printed blank Formlabs flexible polymer lies around $-0.83^{\circ} \mathrm{C}$, which agrees with the common $T_{\mathrm{g}}$ values for poly(methyl acrylate), which can range from $-4^{\circ} \mathrm{C}$ to $20^{\circ} \mathrm{C}$ [21]. Even though the overall shape of the DSC curve was similar for different GO/Formlabs flexible nanocomposites and pure Formlabs flexible polymer (Fig. 5), the increase in the added GO concentration results in a slight increase in the glass transition temperature (which was within experimental measurement error) of the 3D printed $\mathrm{GO} /$ Formlabs flexible composites as shown in Fig. 5. This trend holds true for 0.1 and $0.3 \mathrm{wt}-\%$ GO samples with $T_{\mathrm{g}}$ values of $-0.76^{\circ} \mathrm{C}$ and $2.84^{\circ} \mathrm{C}$ respectively. At $0.2 \mathrm{wt}-\%$ $\mathrm{GO}$, the $T_{\mathrm{g}}$ deviates from the observed behavior, with $T_{\mathrm{g}}$ in fact increasing to the value of around $-8.7^{\circ} \mathrm{C}$. The aforementioned, high degree of agglomeration in the $3 \mathrm{D}$ printed $0.2 \mathrm{wt}-\%$ GO sample (Fig. S1), leads to an interruption of photo-curing, resulting in a decrease in photo-crosslinking. By comparing DSC curves of $\mathrm{GO} /$ Formlabs flexible composites in current work with those in others SLA or DLP printed $\mathrm{GO} /$ polymer composites studies [16], a similar pattern was observed, where the increase in GO concentration resulted in the decrease of glass transition temperature of the composite.

The TGA showed a slight change in the thermal decomposition behavior of the samples, as shown in

Table $1 T_{\mathrm{g}}$-DSC result of the GO/Formlabs flexible composite with different GO content based on the Fig. 5

\begin{tabular}{lc}
\hline Sample & $T_{\mathrm{g}} /{ }^{\circ} \mathrm{C}$ \\
\hline Blank & -0.83 \\
$0.1 \mathrm{wt}-\%$ GO & -0.76 \\
$0.2 \mathrm{wt}-\%$ GO & -8.70 \\
$0.3 \mathrm{wt}-\%$ GO & 2.84 \\
\hline
\end{tabular}

Fig. 6(a). It is apparent that all the samples are decomposed in two steps based on the differential TGA (DTGA) spectrum as shown in Fig. 6(b), with the first mass loss attributed to partial loss of the side groups and the second one to the depolymerisation of the polymer. Both decomposition steps are gradually shifted to the slightly lower decomposition temperatures, with the increase in GO concentration in $3 \mathrm{D}$ printed nanocomposites, compared to the blank Formlabs flexible polymer. These observations suggest that thermal stability of the Formlabs flexible polymer is slightly reduced upon addition of GO.

The mechanical properties of the 3D printed $\mathrm{GO} /$ Formlabs flexible nanocomposites are in shown Fig. 7 and summarized in Table 2. The results showed that compared to the 3D printed blank Formlabs flexible polymer, the mechanical strength of the nanocomposites decreased upon the GO addition into the raw flexible resin. The ultimate tensile strength of the $0.1 \mathrm{wt}-\% \mathrm{GO} /$ Formlabs flexible nanocomposite $(1.3 \mathrm{MPa})$ was almost half of that of the 3D printed blank flexible polymer $(\sim 2.5 \mathrm{MPa})$, while composites with GO concentrations of $0.2 \mathrm{wt}-\%$ (1 MPa) and 0.3 wt-\% (1.2 MPa) were $59 \%$ and $52 \%$ lower respectively. The Young's Modulus of 3D printed polymer nanocomposites decreased gradually from around 9.63 to 5.43 when the GO content increased gradually from 0 to

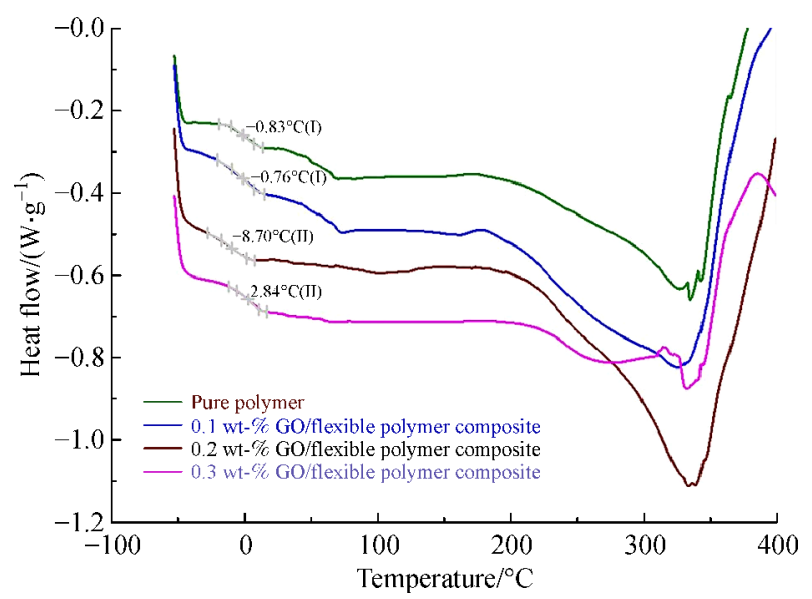

Fig. 5 DSC spectrum $\left(-100^{\circ} \mathrm{C}-400^{\circ} \mathrm{C}\right)$ of the $\mathrm{GO} /$ Formlabs flexible nanocomposite vs. pure Formlabs flexible polymer $3 \mathrm{D}$ MPSL structure. 

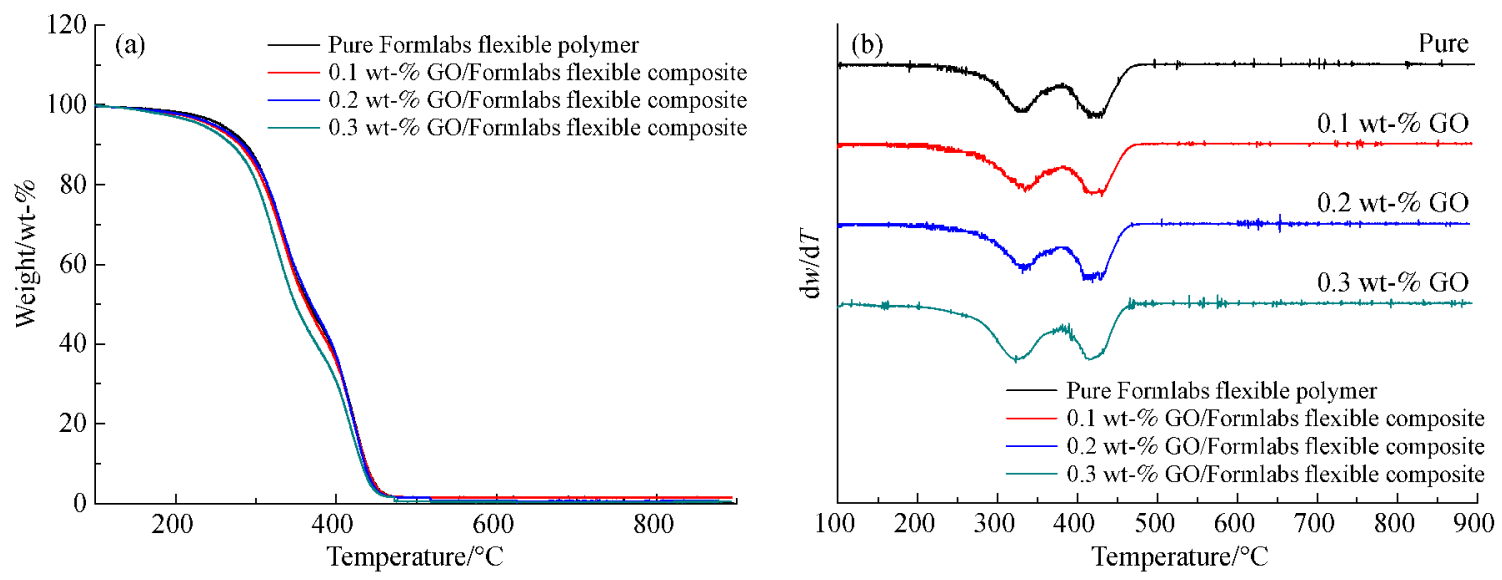

Fig. 6 (a) TGA, and (b) DTGA spectrum of the GO/resin 3D MPSL structure vs. pure resin structure in $100^{\circ} \mathrm{C}-900^{\circ} \mathrm{C}$.

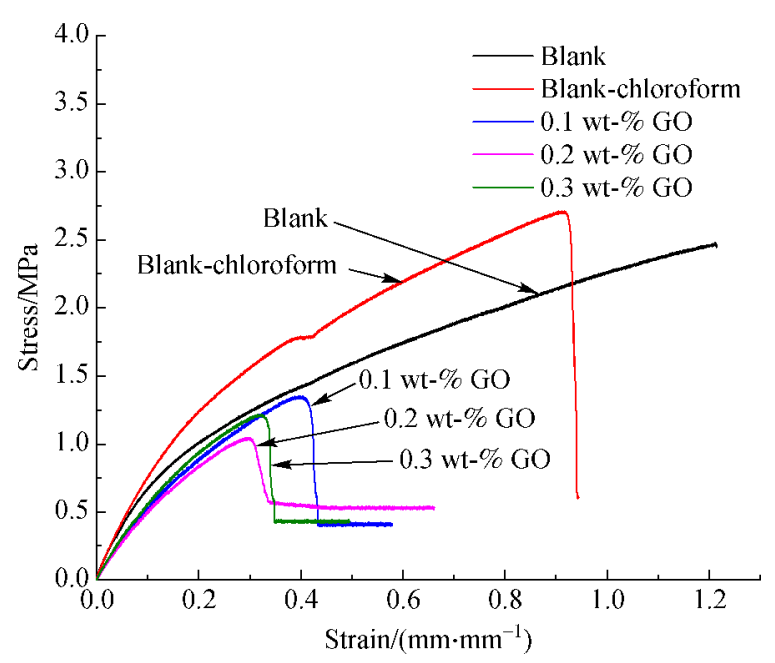

Fig. 7 Tensile test of the 3D printed GO nanocomposites with different GO loadings.

$0.2 \mathrm{wt}-\%$, but rebounded back to around 6.2 when the GO content further increased to $0.3 \mathrm{wt}-\%$. The incorporation of GO has also adversely affected the already limited elongation of Formlabs flexible polymer, with elongation at break significantly decreased from $120 \%$ to around $30 \%$ when the GO content was increased from 0 to $0.3 \mathrm{wt}-\%$. The deviation at $0.2 \mathrm{wt}-\%$ GO from the trend in case of the mechanical properties, was also observed in the thermal properties as was mentioned previously in the DSC analysis (Fig. 5). From the visual observation of the 3D printed GO/Formlabs flexible composites, as well as the SEM and TEM images, it was evident that GO nanosheets tended to agglomerate within the polymer matrix, instead of being uniformly distributed. The presence of GO flakes within the polymer matrix can result in localized stress concentrations, which would in turn lead to premature failure. This could explain the deterioration of mechanical properties upon the inclusion of GO within the polymer matrix in this work. The tendency of the stress variation upon increase in the GO content was different from the reported works synthesized by traditional method $[13,15]$, which was irregular to the trend of the GO content in the raw GO/Formlabs flexible resin. Whilst according to Chiappone et al. [20] who employed SLA-based printing technique to 3D print GO/PEGDA nanocomposite, the higher loadings of GO ( $>0.5 \mathrm{phr}$ ) result in decrease of mechanical properties, due to a negative influence on the photocuring kinetics. The general trend observeddecrease in tensile strength and elongation at break with an increase in GO concentration, can be explained by the fact that at higher GO concentrations, the nanosheets tend to aggregate, which decreases cross-link sites and disrupt the polymer chain orientations at high strain values [22]. From the mechanical test (Fig. 7) 0.2 wt-\% GO nanocomposite (tensile strength $=1.2 \mathrm{MPa}$ and elongation at break $=32 \%$ ) did not show any significant variation compared to $0.3 \mathrm{wt}-\% \mathrm{GO}$ (tensile strength $=1 \mathrm{MPa}$ and elongation at break $=30 \%$ ). However, the higher degree of agglomeration in the $3 \mathrm{D}$ printed $0.2 \mathrm{wt}-\%$ GO sample, as illustrated by the optical microscope image (Fig. S1), would explain the slight deviation in mechanical properties. The DSC characterization showed that the glass transition temperature $\left(T_{\mathrm{g}}\right)$ was generally increased when the amount of GO increased from $0 \mathrm{wt}-\%$ (pure polymer) to $0.3 \mathrm{wt}-\%$ in the polymer composite, but it was abnormally reduced when the GO content was $0.2 \mathrm{wt}-\%$ in the composite. The aforementioned, high degree of agglomeration in the 3D printed $0.2 \mathrm{wt}-\%$ GO sample (Fig. S1), leads to an interruption of photo-curing, resulting in a decrease in photo-crosslinking. Further works on the improvement of the mechanical properties of the $\mathrm{GO} /$ Formlabs flexible composites after the 3D printing process such as thermal annealing under vacuum will be the expected tasks in future.

In the work carried out by Manapat et al. [16], it was also observed that the increase in GO content resulted in a decrease in the mechanical strength, which they attributed to higher concentration of GO leading to excessive 
Table 2 List of the Young's Modulus of the 3D printing GO/Formlabs flexible composites with different GO contents

\begin{tabular}{lccc}
\hline 3D Printed structures & Young's Modulus /MPa & Elongation at break /\% & Ultimate tensile strength /MPa \\
\hline Pure Formlabs flexible polymer & 9.64 & 120 & 2.5 \\
Formlabs flexible polymer + Chloroform & 8.82 & 90 & 2.7 \\
0.1 wt-\% GO/Formlabs flexible composite & 5.77 & 40 & 1.3 \\
0.2 wt-\% GO/Formlabs flexible composite & 5.43 & 30 & 1 \\
0.3 wt-\% GO/Formlabs flexible composite & 6.18 & 32 & 1.2 \\
\hline
\end{tabular}

interplatelet interaction instead of GO-resin hydrogen bonding, thus decreasing GO-resin interaction, as well as the fact that the presence of GO in the resin can lower the efficiency of photopolymerization because the filler can serve as a barrier or hindrance to incoming laser light [16]. This observation was also similar to the case of carbon nanotube reinforced poly(methyl methacrylate) [23] and the GO containing PEGDA [20], respectively. Another possible explanation offered by Manapat et al. [16] is that $\mathrm{GO}$ is acting as a chain transfer agent, which inhibits the further growth of polymer chain. When examining the morphology of the as-synthesized GO using atomic force microscopy, Manapat et al. [16] also observed the presence of wrinkles, in the GO sheets, which could affect the stress distribution on the surface of GO, which would hinder the interlayer adhesion between $\mathrm{GO}$ and the resin, resulting in poor load transfer, which consequently would result in lower mechanical strength.

To alleviate this problem, certain post-processing steps can be employed. For instance, Manapat et al. [16]. enhanced thermomechanical properties of SLA fabricated GO nanocomposites via a simple mild annealing process $\left(100^{\circ} \mathrm{C}\right.$ for $\left.12 \mathrm{~h}\right)$, which resulted in drastic increase in mechanical properties with the highest percent increase recorded at $673.6 \%$ for the $1 \mathrm{wt}-\%$ GO nanocomposite. The assumptions used to explain these phenomena, were verified through spectroscopic techniques by Manapat et al. and can be summed up as follows: (1) There is lower defect density with increasing annealing temperature; (2) There is enhanced cross-linking between GO and the resin at $100^{\circ} \mathrm{C}$ due to acid-catalyzed, esterification, and (3) The sample lost intercalated water in its structure at $100^{\circ} \mathrm{C}$ and hence no mass loss was observed in this region.

Another approach involves surface functionalization of GO prior to the addition to photosensitive resin. Li et al. [17] recently demonstrated that by grafting 2-hydroxyethyl methacrylate groups to the surface of the GO, improved dispersibility and interfacial compatibility of GO nanosheets with the photosensitive resin can be achieved.

Up to date, there was still no report on direct fabrication of GO modified elastomers via SLA. Further studies are expected in future for the more detailed information on such composites. These include the improvement of the mechanical strength of the 3D printed GO/elastomer composites by thermal annealing of 3D printed samples as was demonstrated by Manapat et al. [16], and exploring effective approaches for the uniform distribution of GO in the photoactive SLA resins for improving the quality products with enhanced thermal and mechanical properties. However, the relatively simple pathway of the GO/ polymer gel synthesis via the direct mixing of the GO dispersion with commercial SLA resin in one-step process demonstrated in current study provided the new gateway for the convenient preparation of the GO/elastomer nanocomposites via SLA printing in future.

\section{Conclusions}

Notably, it was demonstrated for the first time that GO loadings can be incorporated into commercial "flexible" photoresins to successfully fabricate $\mathrm{GO}$ /elastomer nanocomposites via readily accessible, consumer-oriented SLA printer in a user-friendly manner. The inclusion of GO, with concentrations of up to $0.3 \mathrm{wt}-\%$, to the commercially available SLA resin, composed of mixture of acrylated oligomers and acrylated monomers, resulted in a successful fabrication of $\mathrm{GO} /$ elastomer nanocomposites using a bottom-up SLA approach. Despite the successful incorporation of GO, both the mechanical strength and stiffness (Young's Modulus), as well as the elongation of the resulting polymer decreased with the addition of GO. The thermal properties were also adversely affected upon the increase in the GO content based on DSC and TGA results. It was proposed that the non-uniform dispersion of GO within the SLA resin, causing large GO agglomeration within the $3 \mathrm{D}$ printed composites, can significantly change both mechanical and thermal properties of the resulting nanocomposites. Further in-depth investigations on effective approaches to achieve uniform GO dispersions in SLA resins, as well as the annealing treatment of the $\mathrm{GO} /$ elastomer nanocomposites for mechanical and thermal enhancements were proposed for future development of $3 \mathrm{D}$ printed nanocomposite elastomers.

\footnotetext{
Acknowledgements This work described in this paper was supported by a grant from the Hong Kong-Scotland Partners in Post Doctoral Research Scheme under the Research Grants Council of Hong Kong and the Scotland Government (S-HKU702/15). The research is also supported by the UK Engineering and Physical Sciences Research Council via grant number EP/ R012164/2 and The Royal Society via grant number RSG\R1\180162.
} 
Electronic Supplementary Material Supplementary material is available in the online version of this article at https://doi.org/10.1007/s11705-019$1836-\mathrm{x}$ and is accessible for authorized users.

Open Access This article is licensed under a Creative Commons Attribution 4.0 International License, which permits use, sharing, adaptation, distribution and reproduction in any medium or format, as long as you give appropriate credit to the original author(s) and the source, provide a link to the Creative Commons licence, and indicate if changes were made. The images or other third party material in this article are included in the article's Creative Commons licence, unless indicated otherwise in a credit line to the material. If material is not included in the article's Creative Commons licence and your intended use is not permitted by statutory regulation or exceeds the permitted use, you will need to obtain permission directly from the copyright holder. To view a copy of this licence, visit http://creativecommons.org/licenses/by/4.0/.

\section{References}

1. Lin D, Jin S Y, Zhang F, Wang C, Wang Y Q, Zhou C, Cheng J. 3D stereolithography printing of graphene oxide reinforced complex architectures. Nanotechnology, 2015, 26(43): 434003

2. Zhu Y W, Murali S, Cai W W, Li X S, Suk J W, Potts J R, Ruoff R $\mathrm{S}$. Graphene and graphene oxide: Synthesis, properties, and applications. Advanced Materials, 2010, 22(35): 3906-3924

3. Lee C, Wei X, Kysar J W, Hone J. Measurement of the elastic properties and intrinsic strength of monolayer graphene. Science, 2008, 321(5887): 385-388

4. Balandin A A, Ghosh S, Bao W, Calizo I, Teweldebrhan D, Miao F, Lau C N. Superior thermal conductivity of single-layer graphene. Nano Letters, 2008, 8(3): 902-907

5. Kuilla T, Bhadra S, Yao D H, Kim N H, Bose S, Lee J H. Recent advances in graphene based polymer composites. Progress in Polymer Science, 2010, 35(11): 1350-1375

6. Verdejo R, Bernal M M, Romasanta L J, Lopez-Manchado M A. Graphene filled polymer nanocomposites. Journal of Materials Chemistry, 2011, 21(10): 3301-3310

7. Korhonen H, Sinh L H, Luong N D, Lehtinen P, Verho T, Partanen J, Seppala J. Fabrication of graphene-based 3D structures by stereolithography. Physica Status Solidi. A, Applications and Materials Science, 2016, 213(4): 982-985

8. Papageorgiou D G, Kinloch I A, Young R J. Graphene/elastomer nanocomposites. Carbon, 2015, 95: 460-484

9. Iliut M, Silva C, Herrick S, McGlothlin M, Vijayaraghavan A. Graphene and water-based elastomers thin-film composites by dipmoulding. Carbon, 2016, 106: 228-232

10. Al-Saygh A, Ponnamma D, AlMaadeed A M, Vijayan P P, Karim A, Hassan M. Flexible pressure sensor based on PVDF nanocomposites containing reduced graphene oxide-titania hybrid nanolayers. Polymers, 2017, 9(12): 33

11. He Y, Li W, Yang G L, Liu H, Lu J Y, Zheng T T, Li X J. A novel method for fabricating wearable, piezoresistive, and pressure sensors based on modified-graphite/polyurethane composite films. Materials (Basel), 2017, 10(7): 684

12. Xing W, Li H, Huang G, Cai L H, Wu J. Graphene oxide induced crosslinking and reinforcement of elastomers. Composites Science and Technology, 2017, 144: 223-229

13. Zhang C, Zhai T, Dan Y, Turng L S. Reinforced natural rubber nanocomposites using graphene oxide as a reinforcing agent and their in situ reduction into highly conductive materials. Polymer Composites, 2017, 38(S1): E199-E207

14. Li X D, Honari M M, Fu Y Y, Kumar A, Saghlatoon H, Mousavi P, Chung H J. Self-reinforcing graphene coatings on 3D printed elastomers for flexible radio frequency antennas and strain sensors. Flexible and Printed Electronics, 2017, 2(3): 035001

15. Li S, Li Z, Burnett T L, Slater T J A, Hashimoto T, Young R J. Nanocomposites of graphene nanoplatelets in natural rubber: Microstructure and mechanisms of reinforcement. Journal of Materials Science, 2017, 52(16): 9558-9572

16. Manapat J Z, Mangadlao J D, Tiu B D B, Tritchler G C, Advincula R C. High-strength stereolithographic 3D printed nanocomposites: Graphene oxide metastability. ACS Applied Materials \& Interfaces, 2017, 9(11): 10085-10093

17. Li J H, Wang L F, Dai L J, Zhong L P, Liu B, Ren J H, Xu Y F. Synthesis and characterization of reinforced acrylate photosensitive resin by 2-hydroxyethyl methacrylate-functionalized graphene nanosheets for 3D printing. Journal of Materials Science, 2018, 53 (3): $1874-1886$

18. Thrasher C J, Schwartz J J, Boydston A J. Modular elastomer photoresins for digital light processing additive manufacturing. ACS Applied Materials \& Interfaces, 2017, 9(45): 39708-39716

19. Kovtyukhova N I, Ollivier P J, Martin B R, Mallouk T E, Chizhik S A, Buzaneva E V, Gorchinskiy A D. Layer-by-layer assembly of ultrathin composite films from micron-sized graphite oxide sheets and polycations. Chemistry of Materials, 1999, 11(3): 771-778

20. Chiappone A, Roppolo I, Naretto E, Fantino E, Calignano F, Sangermano M, Pirri F. Study of graphene oxide-based 3D printable composites: Effect of the in situ reduction. Composites. Part B, Engineering, 2017, 124: 9-15

21. Pethrick R A. Polymer Structure Characterization: From Nano to Macro Organization in Small Molecules. Cambridge: The Royal Society of Chemistry Publishing, 2014, 254

22. Lee S K, Kim B K. Synthesis and properties of shape memory graphene oxide/polyurethane chemical hybrids. Polymer International, 2014, 63(7): 1197-1202

23. Maurice G, Rouxel D, Vincent B, Hadji R, Schmitt J F, Taghite M, Rahouadj R. Investigation of elastic constants of polymer/ nanoparticles composites using the Brillouin spectroscopy and the mechanical homogenization modeling. Polymer Engineering and Science, 2013, 53(7): 1502-1511 